\title{
Scaling Law for Liquid Splashing Inside a Container Drop Impact on a Solid Surface
}

\author{
Bohua Sun ${ }^{1}$ \\ ${ }^{1}$ Cape Peninsula University of Technology, Cape Town, South Africa \\ sunb@cput.ac.za
}

(Dated: November 14, 2018)

\begin{abstract}
This letter attempts to find splashing height of liquid-filled container drop impact to a solid surface by dimensional analysis (DA). Two solutions were obtained by both traditional DA and directed DA without solving any governing equations. It is found that the directed DA can provide much more useful information than the traditional one. This study shows that the central controlling parameter is called splash number $\mathrm{Sp}=\mathrm{GaLa}^{\beta}=\left(\frac{g R^{3}}{\nu^{2}}\right)\left(\frac{\sigma R}{\rho \nu^{2}}\right)^{\beta}$, which is the collective performance of each quantity. The splash height is given by $\frac{h}{H}=\left(\frac{\rho \nu^{2}}{\sigma R}\right)^{\alpha} f\left[\frac{g R^{3}}{\nu^{2}}\left(\frac{R \sigma}{\rho \nu^{2}}\right)^{\beta}\right]=\frac{1}{\mathrm{La}} f\left(\mathrm{Ga} \cdot \mathrm{La}^{\beta}\right)$. From the physics of the splashing number, we can have a fair good picture on the physics of the liquid splashing as follows: the jets propagation will generate vortex streets from the container bottom due to sudden pressure increasing from drop impact (water-hammer effect), which will travel along the container sidewall to the centre of the container and subsequently excite a gravity wave on the liquid surface. The interaction between the gravitational force, surface force and viscous force is responsible for creating droplet splash at the liquid surface.
\end{abstract}

Keywords: Liquid splashing, dimensional analysis, directed dimensional analysis

\section{INTRODUCTION}

Liquid splashing can be happening anytime and anywhere [1-6], from water-cup dropping to SpaceX rocket returning (shown in Figure 1 and 2). For rocket returning, the study of liquid splashing is useful on rocket stabilization, if there were some fuel still left. Free falling liquid drop impact to a solid surface has been studied well [4-6], however the liquid splashing inside a container has little results.

Without solving governing equations, this study attempts to attack the splashing system by using dimensional analysis. The most powerful use of dimensional analysis is to predict the outcome of an numerical experiment, depending on the variables, whilst providing theoretical insight. Dimensional analysis may come across as simply trying to fit pieces of a puzzle together by trial and error. However, identifying the quantities that are relevant for a given problem is a demanding task, which requires deep physical insight. This may be done as follows: make a list of all quantities on which the answer must

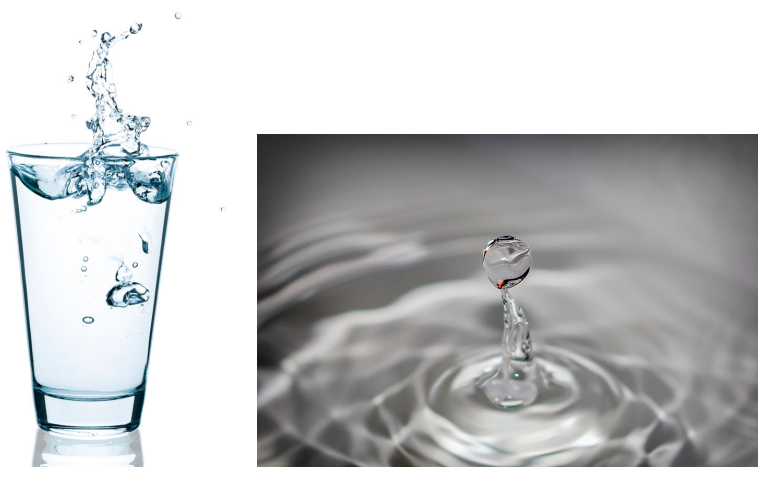

Figure 1: Water splash

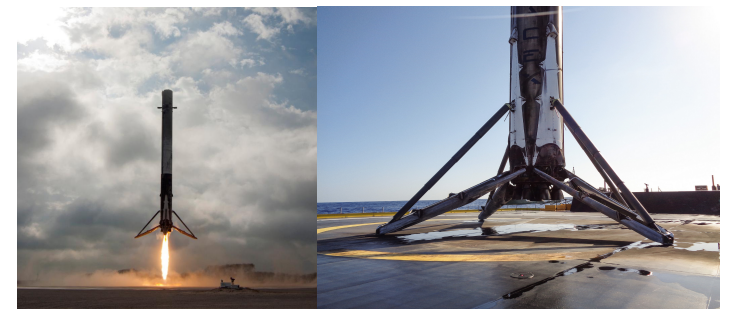

Figure 2: SpaceX rocket return

depend, then write down the dimensions of these quantities, and finally demand that these quantities should be combined into a functional form that provides the right dimension [7-12].

This article attempts to investigation the problem by using dimensional analysis (DA). To make the paper selfcontained, the paper is organised as follows. After this introduction, in Section 2 the traditional dimensional analysis (DA) is used and first proposal on scaling law is formulated; in Section 3, the directed dimensional analysis (DDA) is applied, and a more meaningful scaling law is obtained. In Section 4 the discussions on the obtained scaling laws have been proposed. Finally, section 5 concludes the paper with perspectives.

\section{SCALING LAW FORMULATION BY TRADITIONAL DIMENSIONAL ANALYSIS}

Giving a liquid container shown in Figure 3, containing liquid with density $\rho$, kinematic viscosity $\nu$, surface tension $\sigma$, drop impact to the solid surface from height $H$.

The liquid container dropping can be roughly divided into two stages. Stage 1: From height $H$ to the solid 


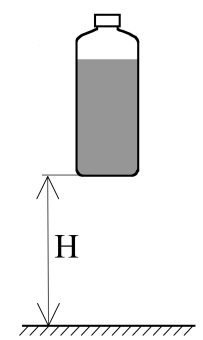

Figure 3: Liquid-filled container drop impact to a solid surface from height $H$

Table I: List of quantities and their dimensions

\begin{tabular}{c|c|c|c|c|c|c}
\hline$h$ & $R$ & $g$ & $\rho$ & $\nu$ & $\sigma$ & $H$ \\
\hline$L$ & $L$ & $L T^{-2}$ & $M L^{-3}$ & $L^{2} T^{-1}$ & $M T^{-2}$ & $L$ \\
\hline
\end{tabular}

surface, since the liquid is dropping together with the container under the gravitation, there is no splashing [18]. Stage 2: Once the container dropped to the solid surface, the impact impulse will apply to the container bottom and then create jets that originate from bottom. If the container is not full with the liquid, liquid splashing must be happen due to the existing of free liquid surface.

Assuming the liquid container is rigid with characteristic length scale $R$, which can be the radius for circular container, to find the maximum splash height $h$.

List of quantities and their dimensions in DDA are shown in Table I. The splash height $h$ must be the function of other 7 quantities

$$
h=f(R, g, \rho, \mu, \sigma, H) .
$$

The problem has 7 quantities in total, uses only 3 units or dimensions, namely mass $\mathrm{M}$, length $\mathrm{L}$ and time $\mathrm{T}$. According to the Buckingham $\Pi$ theorem [7], therefore should have four $\Pi_{i}$. We can choose $R, \nu, \rho$ as basic quantities, the problem can be rewritten as dimensionless form: $\Pi_{1}=f\left(\Pi_{2}, \Pi_{3}, \Pi_{4}\right)$, where $\Pi_{1}=\frac{h}{H}, \Pi_{2}=\frac{H}{R}$, $\Pi_{3}=\frac{g R^{3}}{\nu^{2}}, \Pi_{4}=\frac{\sigma R}{\rho \nu^{2}}$.

Replace the $\Pi_{3}$ and $\Pi_{4}$ by the Galileo number Ga and the Laplace number La, respectively, namely $\mathrm{Ga}=\frac{g R^{3}}{\nu^{2}}$ and $\mathrm{La}=\frac{\sigma R}{\rho \nu^{2}}$, the scaling law of the liquid splashing is given by

$$
h=R f\left(\frac{H}{R}, \mathrm{Ga}, \mathrm{La}\right)=R f\left(\frac{H}{R}, \mathrm{Ga}, O h^{-2}\right) .
$$

The Galileo number ( $\mathrm{Ga}$ ) is a dimensionless number named after Italian scientist Galileo Galilei (1564-1642). It may be regarded as proportional to gravity forces divided by viscous forces. The Laplace number (La) is a dimensionless number used in the characterization of free surface fluid dynamics. It represents a ratio of surface tension to the momentum-transport inside a fluid. The Ohnesorge number $(\mathrm{Oh})$ is a dimensionless number that relates the viscous forces to inertial and surface ten-
Table II: List of quantities and their dimensions in DDA

\begin{tabular}{c|c|c|c|c|c|c}
\hline$h$ & $R$ & $g$ & $\rho$ & $\nu$ & $\sigma$ & $H$ \\
\hline$L_{z}$ & $\left(L_{x} L_{y}\right)^{1 / 2}$ & $L_{z} T^{-2}$ & $M\left(L_{x} L_{y} L_{z}\right)^{-1}$ & $\left(L_{x} L_{y}\right)^{3 / 4} L_{z}^{1 / 2} T^{-1}$ & $M T^{-2}$ & $L_{z}$ \\
\hline
\end{tabular}

sion forces. The number was defined by Wolfgang von Ohnesorge in his 1936 doctoral thesis [13].

It should be noted here that the relation formulated in Eq.(2) can not give us much information, we will revisit the problem by using the directed dimensional analysis (DDA).

\section{SCALING LAW FORMULATION BY DIRECTED DIMENSIONAL ANALYSIS}

The scaling law in Rq.(2) can still be simplified if we apply directed dimensional analysis (DDA) [14-16]. Set a $x y z$ coordinate system, taking into account their directions, accordingly the length dimensions are denoted by $L_{x}, L_{y}, L_{z}$.

Assuming the container drops from height $H$ along $z$ direction, its dimension is denoted by $\operatorname{dim}(H)=$ $L_{z}$. Accordingly, the gravitation acceleration $\operatorname{dim}(g)=$ $L_{z} T^{-2}$, density $\operatorname{dim}(\rho)=M L_{x}^{-1} L_{y}^{-1} L_{z}^{-1}$, surface tension $\operatorname{dim}(\sigma)=M T^{-2}$, the container characteristic length $\operatorname{dim}(R)=L_{x}^{1 / 2} L_{y}^{1 / 2}$, the splashing height $\operatorname{dim}(h)=L_{z}$. For directed dimensional analysis, the difficulty is how to define the kinematic viscosity $\nu$ in the DDA. Since the dimension should be uniformly in all direction, and take into account surface feature of the viscosity, then the kinematic viscosity $\nu$ can be redefined as follows: $\operatorname{dim}(\nu)=L^{2} T^{-1}=L_{x}^{3 / 4} L_{y}^{3 / 4} L_{z}^{1 / 2} T^{-1}$.

List of quantities and their dimensions in DDA are shown in Table II.

Within the directed dimensional analysis (DDA), five dimensions, $M, T, L_{x}, L_{y}, L_{z}$, are used, namely, $j=5$. The total number of quantities are still $n=7$, therefore the number of dimensionless $\Pi$ will be reduced from four to two, which is calculated from $n-j=7-5$, which can make the final result in a much simpler format. We can choose $R, H, \rho, \sigma, \nu$ as basic quantities, thus the two dimensionless $\Pi_{1}$ and $\Pi_{2}$ are derived as follows

$$
\Pi_{1}=\frac{h}{H}\left(\frac{\sigma R}{\rho \nu^{2}}\right)^{\alpha}=\frac{h}{H} \mathrm{La}^{\alpha}
$$

and

$$
\Pi_{2}=\frac{g R^{3}}{\nu^{2}}\left(\frac{\sigma R}{\rho \nu^{2}}\right)^{\beta}=\mathrm{GaLa}^{\beta},
$$

From the Buckingham theorem [7, 9], the universal relation of splashing is given by $\Pi_{1}=f\left(\Pi_{2}\right)$, namely

$$
\frac{h}{H}=\left(\frac{\rho \nu^{2}}{\sigma R}\right)^{\alpha} f\left[\frac{g R^{3}}{\nu^{2}}\left(\frac{R \sigma}{\rho \nu^{2}}\right)^{\beta}\right]=\frac{1}{\mathrm{La}^{\alpha}} f\left(\mathrm{Ga} \cdot \mathrm{La}^{\beta}\right),
$$

where both $\alpha$ and $\beta$ can not be determined only by the DDA, which can be viewed as adjustable parameters. 
It is surprise to see that the result in Eq (5) by the DDA is totally different from the one in Eq (2) by the DA. Furthermore, the DDA in Eq. (5) is much simpler and physically meaningful, which reveals that the splashing height is proportional to the dropping height and the function of product $\mathrm{Ga} \cdot \mathrm{La}^{\beta}$.

\section{DISCUSSIONS}

Although we can not finalise the format of Eq.(5) by only directed dimensional analysis (DDA), the relation derived from the DDA can still provide us a vital information even without solving governing equations. This is the beauty of the DA, namely it can identify the most crucial quantities combinations before we are even going to do either numerical simulation or experimental test. In this case, the controlling parameter in liquid splashing process is $\frac{g R^{3}}{\nu^{2}} \frac{\sigma R}{\rho \nu^{2}}=$ GaLa. It means that the five quantities control the splashing process together via their combination $\frac{g R^{3}}{\nu^{2}} \frac{\sigma R}{\rho \nu^{2}}$ rather than in individual. This discovery is important for data fitting of liquid splashing, for future use we define the single combination as splash number (Sp) by

$$
\mathrm{Sp}=\frac{g R^{3}}{\nu^{2}}\left(\frac{R \sigma}{\rho \nu^{2}}\right)^{\beta} .
$$

Therefore, we have the maximum splashing height as follows

$$
\frac{h}{H}=\frac{1}{\mathrm{La}^{\alpha}} f(\mathrm{Sp}) .
$$

The function $f(:)$ can be determined from a test datafitting.

We can set both $\alpha$ and $\beta$ to be some particular values to get a simpler relationship. For instance, without loss generality, we can try following cases.

Case 1: let $\alpha=0$ and $\beta=0$, we have

$$
\Pi_{1}=\frac{h}{H}, \quad \Pi_{2}=\frac{g R^{3}}{\nu^{2}}=\mathrm{Ga} .
$$

The liquid splashing height is therefore given by

$$
\frac{h}{H}=f\left(\frac{g R^{3}}{\nu^{2}}\right)=f(\mathrm{Ga})
$$

This equation is valid for very weak surface tension liquid.

Case 2: let $\alpha=0$ and $\beta=1$, we have

$$
\Pi_{1}=\frac{h}{H}, \quad \Pi_{2}=\frac{g R^{3}}{\nu^{2}} \frac{\sigma R}{\rho \nu^{2}}=\text { GaLa. }
$$

The liquid splashing height is therefore given by

$$
\frac{h}{H}=f\left(\frac{g R^{3}}{\nu^{2}} \frac{\sigma R}{\rho \nu^{2}}\right)=f(\mathrm{GaLa})=F\left(\frac{\mathrm{Ga}}{\mathrm{Oh}^{2}}\right) .
$$

Case 3: let $\alpha=1$ and $\beta=0$, we have

$$
\Pi_{1}=\frac{h}{H}\left(\frac{\sigma R}{\rho \nu^{2}}\right), \quad \Pi_{2}=\frac{g R^{3}}{\nu^{2}}=\mathrm{Ga} .
$$

The liquid splashing height is therefore given by

$$
\frac{h}{H}=\frac{\rho \nu^{2}}{\sigma R} f\left(\frac{g R^{3}}{\nu^{2}}\right)=\frac{1}{\mathrm{La}} f(\mathrm{Ga}) .
$$

Case 4: let $\alpha=1$ and $\beta=-1$, we have

$$
\Pi_{1}=\frac{h}{H}\left(\frac{\sigma R}{\rho \nu^{2}}\right), \quad \Pi_{2}=\frac{\rho g R^{2}}{\sigma}=f(\mathrm{Bo}) .
$$

where the Bond number Bo $=\frac{\rho g R^{2}}{\sigma}$. The liquid splashing height is therefore given by

$$
\frac{h}{H}=\frac{\rho \nu^{2}}{\sigma R} f\left(\frac{\rho g R^{2}}{\sigma}\right)=\frac{1}{\mathrm{La}} f(\mathrm{Bo}) .
$$

From Eq.(5), let's see what is the physics behind splashing. Since the Galileo number (Ga), the Laplace number (La), Ohnesorge number (Oh) and the Bond number (Bo) can be understood as follows

$$
\mathrm{Ga} \sim \frac{\text { gravity forces }}{\text { viscous forces }}
$$

and

$$
\text { La } \sim \frac{\text { surface tension }}{\text { the momentum - transport inside a fluid }},
$$

and

$$
\mathrm{Oh} \sim \frac{\text { viscous forces }}{\sqrt{\text { inertial and surface tension forces }}} .
$$

and

$$
\text { Bo } \sim \frac{\text { area gravity forces }}{\text { surface tension }} .
$$

Based on the general relation Eq.(5), with the help on the understanding of the Galileo number, the Laplace number and the Bond number, we can have a fair good picture on the physics of the liquid splashing as follows: the jets propagation will generate vortex streets from the container bottom due to sudden pressure increasing from drop impact (water-hammer effect), which will travel along the container sidewall to the centre of the container and subsequently excite a gravity wave on the liquid surface. The interaction between the gravitational force and surface force and viscous force is responsible for creating droplet splash at the liquid surface. This mechanism has some kind of similarity with the dragon-wash [17] in ancient China. 


\section{CONCLUSIONS}

Given liquid density $\rho$, kinematic viscosity $\nu$, surface tension $\sigma$, drop height $H$ and the cross-section scale $R$ of the liquid container. In the case of including all these factors, in order to maintain the simplicity of the physical relationship, the splashing height should be taken $h$ in Eq.(15), namely

$$
\frac{h}{H}=\frac{\rho \nu^{2}}{\sigma R} f\left(\frac{\rho g R^{2}}{\sigma}\right)=\frac{1}{\mathrm{La}} f(\mathrm{Bo}),
$$

where the function $f(:)$ can be determined by experiments.

\section{Acknowledgement}

The author wishes to thank Michael Sun of Bishops Diocesan College, Cape Town, for taking pictures and slow-motion videos.
[1] Worthington, A. M. On impact with a liquid surface. Proc. R. Soc. London 34, 217-230 (1882).

[2] Worthington, A. M. \& Cole, R. S. Impact with a liquid surface studied by the aid of instantaneous photography. Paper II. Philos. Trans. R. Soc. London Ser. A, Contain. Pap. a Math. or Phys. Character 194, 175-199 (1900).

[3] Rein, M. Phenomena of liquid drop impact on solid and liquid surfaces. Fluid Dyn. Res. 12, 61-93 (1993).

[4] Yarin, A. L. Drop impact dynamics: Splashing, spreading, receding, bouncing... Annu. Rev. Fluid Mech. 38, 159-192 (2006).

[5] Gekle S., Gordillo J. M., van der Meer D., Lohse D. Highspeed jet formation after solid object impact. Phys. Rev. Lett. 102, 034502 (2009).

[6] Josserand, C., Thoroddsen, S. Drop impact on a solid surface. Annual Review of Fluid Mechanics, Annual Reviews, 2016, 48, pp.365-391.

[7] Buckingham, E., Phil. Mag. 42, 696-719 (1921).

[8] Hecksher, T., Insights through dimensions, Nature Physics, 13, 2017.

[9] Sun, B., Dimensional Analysis and Lie Group (In Chinese). China High Education Press, Bejing (2016)

[10] Sun, B., Incompatible deformation field and Riemann curvature tensor. Applied Mathematics and Mechanics, 38(3), 311-332 (2017) https://doi.org/10.1007/s10483$017-2176-8$

[11] Sun, B., Capillary wrinkling scaling laws of floating elastic thin film with a liquid drop, Sci. China-Phys. Mech. Astron. 61, 024721 (2018), https://doi.org/10.1007/s11433-017-9116-5

[12] Sun,B., Kepler's third law of n-body periodic orbits in a Newtonian gravitation field. Sci. China-Phys. Mech. Astron. 61, 054721 (2018)

[13] https://en.wikipedia.org/wiki/List_of_dimensionless_quantities

[14] Huntley H. E. Dimensional Analysis. Dover,1967.

[15] Siano D. Orientational analysis - a supplement to dimensional analysis - Part 1. J. Franklin Institute. 1985,320(320):267.

[16] Siano D. Orientational analysis - tensor analysis and the group properties of the SI supplementary units - Part 2. J. Franklin Institute. 1985, 320(320):285.

[17] Lee, C.B. Experimental studies of surface waves inside a cylindrical container. J. Fluid Mech. (2011), vol. 677, pp. 39-62.

[18] There is splashing if there were no container. 